\section{PSICOLOGÍA IBEROAMERICANA}

Psicología Iberoamericana

ISSN: 1405-0943

psicología.iberoamericana@uia.mx

Universidad Iberoamericana, Ciudad de México

México

Pulido Rull, Marco Antonio; Saavedra Villagómez, Uguette; Gallardo Marín, Diego; Ortega Balderas, Valeria; Rojas Márquez, María Fernanda; Villegas Zazueta, María Fernanda Validez y confiabilidad de dos escalas cortas para medir estrés académico Psicología Iberoamericana, vol. 23, núm. 1, enero-junio, 2015, pp. 28-39 Universidad Iberoamericana, Ciudad de México

Distrito Federal, México

Disponible en: http://www.redalyc.org/articulo.oa?id=133944230004

Cómo citar el artículo

- Número completo

- Más información del artículo

- Página de la revista en redalyc.org

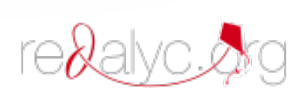

Sistema de Información Científica

Red de Revistas Científicas de América Latina, el Caribe, España y Portugal Proyecto académico sin fines de lucro, desarrollado bajo la iniciativa de acceso abierto 


\title{
Validez y confiabilidad de dos escalas cortas para medir estrés académico
}

\author{
Assessing the validity and reliability of two short scales \\ to gauge academic strees
}

\author{
Marco Antonio Pulido Rull \\ Uguette Saavedra Villagómez \\ UNIVERSIDAD INTERCONTINENTAL \\ Diego Gallardo Marín, Valeria Ortega Balderas \\ María Fernanda Rojas Márquez \\ María Fernanda Villegas Zazueta \\ UNIVERSIDAD IBEROAMERICANA
}

RESUMEN

El propósito de este estudio fue evaluar la validez y confiabilidad de dos escalas cortas que miden estrés académico (SEEU-R y SEEU-RR). Las escalas se aplicaron a una muestra de 997 estudiantes de dos universidades particulares de la Ciudad de México. La validez de constructo se evaluó a través de un análisis factorial exploratorio. La validez empírica del instrumento se evaluó mediante el cálculo de la correlación entre las escalas diseñas, depresión y ansiedad. En general los resultados mostraron que la estructura factorial de las escalas coincide con el fundamento teórico que dio origen al instrumento. La validez empírica se evidenció debido a que las escalas diseñadas mantuvieron correlaciones directas y significativas con ansiedad y depresión. Los resultados se discuten en términos de los usos que se pueden dar a las escalas en contextos universitarios.

Palabras clave: estrés académico, ansiedad, depresión, estudiantes universitarios, validez, confiabilidad.

\section{ABSTRACT:}

This paper seeks to assess the validity and reliability of two short scales that gauge academic stress (SEEU-R and SEEU$R R$ ). The scales were applied to a sample of 997 students from private universities in Mexico City. The construct validity was evaluated through an exploratory factor analysis; the empirical validity of the instrument was assessed by calculating the correlation between designed scales, depression and anxiety. In general the results showed that the scales' factor structure coincided with the theoretical basis that underpinned the instrument. Empirical validity was shown since the designed scales maintained direct and significant correlations with anxiety and depression. The results are discussed in terms of the uses that can be given to scales in university contexts.

Keywords: Academic stress, anxiety, depression, university students, validity, reliability.

Fecha de recepción: 4 de julio de 2014 - Fecha de aceptación: 14 de noviembre de 2014

Los autores desean agradecer al APIEC-UIC y a la Facultad de Psicología de la Universidad Intercontinental las facilidades brindadas para la conducción del presente estudio. Para mayor información relacionada con el trabajo se puede contactar al primer autor en mpulido@uic.edu. 
El concepto de estrés académico es relativamente nuevo,y tal vez pueda considerarse como una evolución de un concepto más viejo: la "ansiedad producida por los exámenes". En su revisión, McDonald (2001) señala que la ansiedad producida por las pruebas, en contextos educativos, inició a finales de la década de los cincuentas. Las variables que la modulan han sido exploradas; adicionalmente, existe un número importante de escalas e instrumentos diseñados para medir el constructo. La investigación sobre ansiedad por exámenes, eventualmente mostró que los estudiantes tienen fuentes de estrés adicionales en las escuelas. Por ejemplo, en sus estudios, Connor $(2001 ; 2003)$ sugirió que las tareas, el trabajo en clase, la presión de los docentes y la competencia con los pares también pueden ser fuentes de estrés para los estudiantes. Así, en ocasiones surgió el concepto de estrés académico; este concepto agrupa diferentes fuentes de estrés que tienen en común el que todas se originan de las actividades educativas que se desarrollan en los planteles (Putwain, 2007).

Al margen de definiciones, tanto la ansiedad producida por los exámenes como el estrés académico han sido estudiados con interés, debido a que ambos constructos se asocian con la ideación suicida en niños y adolescentes (Toero et al., 2001); también en estudiantes universitarios (Gloria \& Ho, 2003; Sue \& Okazaki, 1990). El estrés académico se relaciona con conductas suicidas (Ho, Hong \& Kua, 1999; Ung, 2003). De acuerdo con Chang (2001), el estrés académico también ha sido asociado con déficits cognitivos (por ejemplo dificultades de atención y/o concentración); este hallazgo ha sido replicado por Dyson y Renk (2006) y Edwards, Hershberger, Russell y Markert (2001). El estrés académico también se ha relacionado con hábitos de vida poco saludables; por ejemplo, Hudd et al., (2000) documentaron que estudiantes universitarios que alcanzan altos puntajes de estrés académico también consumen mayores cantidades de comida "chatarra", hacían menos ejercicio y dormían menos. Por su parte, Lumley y Provenzano (2003) encontraron que estudiantes con altos niveles de estrés académico tenían un rendimiento escolar más bajo.

En vista de que el estrés académico en exceso (distrés) puede tener efectos adversos en la salud y formación de los estudiantes, es importante contar con instrumentos psicológicos que permitan evaluarlo de manera confiable y oportuna. Una revisión de la base electrónica Redalyc mostró que existen tres instrumentos de medición redactados en idioma castellano que han sido empleados para medir estrés académico en estudiantes universitarios. García-Ros, PérezGonzález, Pérez-Blasco y Natividad (2012) elaboraron el CEAU, para evaluar estrés académico en estudiantes españoles. En general los resultados mostraron que el instrumento posee propiedades psicométricas aceptables (Nunnally, 1987). A pesar de que los datos muestran que el instrumento es prometedor, la información se obtuvo con una muestra relativamente pequeña (199 estudiantes) y existe evidencia de que los análisis de extracción de factores no son confiables cuando la muestra es menor a 300 individuos (Field, 2005). Además, el instrumento no mide los niveles de estrés académico de los estudiantes, pues tiene por objetivo identificar las actividades escolares que más estresan a los educandos.

Polo, Hernández y Poza (1996), elaboraron un instrumento comparativamente más completo que el de García-Ros y colaboradores (op. cit.): El Inventario de estrés académico (IEA), que mide tanto las actividades escolares que estresan a los estudiantes como los efectos que éstas tienen en los estudiantes. Sin embargo, los datos psicométricos del instrumento se obtuvieron con una muestra de 64 estudiantes. Complementariamente, fuera del análisis de discriminación de reactivos, los autores no llevaron a cabo análisis de validez o de confiabilidad del procedimiento.

Pulido et al., (2011), también elaboraron un instrumento integral; es decir, que evalúa estresores y efectos de éstos sobre el individuo. El inventario sobre estrés en estudiantes universitarios (SEEU) fue aplicado a una muestra de 504 estudiantes universitarios de la Ciudad de México. Los resultados del estudio mostraron validez de constructo y consistencia interna aceptables. Además, a diferencias del CEAU y el IEA, el instrumento de Pulido y colaboradores mide qué tan reciente es el estrés académico y, por tanto, permite saber si el individuo requiere apoyo inmediato. Sin embargo, un problema con el SEEU es que es muy largo (tiene 69 reactivos); además, no ha producido información acerca de su validez empírica. Así pues, el objetivo de esta investigación es el de evaluar las 
propiedades psicométricas de dos versiones cortas del SEEU. Además, en esta investigación se evaluó la validez empírica del SEEU. Específicamente, dado que múltiples investigaciones han mostrado que el estrés académico correlaciona con depresión y ansiedad (Ang \& Huan, 2006; Eremsoy, Celimli \& Gencoz, 2005; Mahmoud Staten, Hall\& Lennie, 2012; Putwain, 2007), en este estudio se evaluó la correlación entre el SEEU y los inventarios de ansiedad y depresión de Beck (BAI y BDI).

\section{MÉTODO}

\section{Participantes}

Hubo en el estudio un total de 997 alumnos de dos universidades particulares de la Ciudad de México. El 53.5\% de la muestra eran mujeres, con una edad promedio de 20.93 años ( $\mathrm{DE}=2.38$ años). La mayoría eran estudiantes de segundo semestre (33.8\%), cuarto (14.6\%), octavo (10.2\%) y tercero (10\%). Predominaron los estudiantes de psicología (24\%), ingenierías (22\%), arquitectura, (21\%) y ciencias de la comunicación (23\%). La mayor parte de los alumnos vivía con su familia (77.5\%).

\section{Instrumentos}

Se emplearon, para reunir los datos, dos versiones cortas del SEEU (Pulido et al., 2011). El inventario original se construyó a partir de la realización de grupos focales conducidos con alumnos de licenciatura. Una vez terminados los grupos focales, la información fue transcrita y estudiada por los autores del trabajo. Se identificaron las principales áreas temáticas y se llevaron a cabo sesiones de lluvia de ideas para generar reactivos que evaluaran cada una de las áreas de interés. Se identificaron 6 áreas de interés: 1) presencia (o ausencia) de síntomas relacionados con el estrés, 2) percepción del estudiante sobre su carga de trabajo académica (y otra), 3) uso de métodos y estrategias para disminuir los efectos desagradables del estrés, 4) efectos del estrés sobre la relación entre el alumno y la institución, 5) efectos del estrés sobre las relaciones interpersonales y 6) la autopercepción del alumno acerca de su eficacia académica. El inventario se contesta con base en una escala de cuatro opciones (últi- mos 30 días, últimos 12 meses, alguna vez y nunca). Complementariamente, para cada pregunta el individuo contesta en una escala de cinco opciones, que permiten evaluar la frecuencia de los eventos de interés (diario, 3 o 4 veces por semana, 1 o 2 veces por semana, algunas veces al mes y algunas veces al año). Pulido et al., (op. cit.) encontraron que la consistencia interna del instrumento fue de .950 . La validez de constructo se determinó a través de un análisis de extracción de factores con una rotación varimax. El análisis factorial identificó seis factores principales (con valores Eigen superiores a 2) que explican el $45 \%$ de la varianza total del instrumento.

En esta investigación el SEEU se recortó, quitando las opciones de respuesta que miden la frecuencia de los eventos (lo cual dejó un total de 35 reactivos). Esta primera versión recortada será denominada en este trabajo como SEEU-R. También se elaboró una versión todavía más corta, utilizando los 18 reactivos con cargas factoriales más altas en el análisis de extracción de factores del SEEU-R. Esta segunda versión reducida será denominada en este trabajo como SEEU-RR.

Se empleó, además, el Inventario de Depresión de Beck (BDI por sus siglas en inglés), estandarizado para la población de la Ciudad de México por Jurado et al. (1998) en una muestra de 1508 habitantes. En su estandarización, la prueba mostró un alfa de Cronbach de .87; señaló adicionalmente validez de constructo y validez concurrente con la Escala de Depresión de Zung. También se empleó el Inventario de Ansiedad de Beck (BAI por sus siglas en inglés). El BAI ha sido evaluado en estudiantes universitarios mexicanos por Tafoya, Gómez, Ortega y Ortiz (2006), en una muestra de 102 individuos. Los investigadores encontraron que el BAI posee validez empírica, ya que diferenció estadísticamente entre individuos con y sin diagnóstico de ansiedad. El BAI también mostró validez concurrente con la Escala de Ansiedad de Hamilton ( $\mathrm{r}=.82)$. En la investigación de Tafoya y colaboradores, citada antes, la consistencia interna del instrumento fue de .86 .

\section{PROCEDIMIENTO}

Los cuestionarios se aplicaron durante los meses de febrero y marzo de 2014. La aplicación se hizo en forma 
grupal, en donde el docente y los alumnos estuvieron de acuerdo en participar. Las instrucciones textuales que recibieron los participantes fueron las siguientes:

"Muchas gracias por su colaboración en este estudio. La finalidad de esta investigación es conocer algunos aspectos de la experiencia universitaria de los estudiantes. Por favor lea con cuidado el siguiente cuestionario y conteste todas las preguntas de la manera más honesta posible. No existen respuestas correctas o incorrectas. Si tiene cualquier duda acerca de las preguntas o las instrucciones le solicitamos que consulte al aplicador. Cuando termine de contestar doble su cuestionario y deposítelo en la urna que se encuentra al frente del salón. Los resultados del presente estudio sólo serán utilizados con fines de investigación. Gracias por su colaboración".

El tiempo aproximado de la aplicación osciló entre los quince y veinte minutos. El aplicador permaneció en el salón durante toda la sesión para contestar dudas y vigilar el proceso. Todos los aplicadores tomaron un curso de una hora antes de incorporarse a este proceso. En todas las universidades muestreadas el proceso de aplicación se llevó a cabo de la misma forma

\section{RESULTADOS}

Una vez codificados, los reactivos fueron procesados mediante el programa SPSS versión 20.0. Con la finalidad de determinar la consistencia interna del instrumento se aplicó una prueba alfa de Cronbach.
Adicionalmente, con el objetivo de obtener el análisis dimensional del instrumento, se llevó a cabo un análisis factorial exploratorio por el método de componentes principales con rotación Varimax. Con la finalidad de seleccionar e interpretar los factores integrantes, se tomó como criterio de elección aquellos valores Eigen iguales o superiores a 1.0. De igual forma, para poder elegir a un reactivo como perteneciente a un factor, se tomó como criterio de selección que su carga factorial fuese de .400 o mayor (y que ésta fuese positiva). En caso de que un reactivo apareciera en más de un factor, se clasificó a éste en aquel factor en el cual su carga factorial fuese más alta). Con base en los criterios anteriores, el instrumento arrojó seis factores que agruparon 35 reactivos y que en conjunto explicaron el 54.06\% de la varianza total. La tabla 1 muestra el número de factor, seguido del número de reactivos, su valor Eigen y los porcentajes de varianza simples y acumulados. La prueba de esfericidad de Bartlett mostró un valor aceptable $(\operatorname{Chi}(595)=12412.85, \mathrm{p}<.001)$, la prueba KMO también mostró un valor aceptable (.948>.5). La rotación alcanzó la convergencia en 25 iteraciones.

La tabla 2 muestra la matriz factorial rotada, resultado del análisis efectuado, e indica aquellos ítems que cumplieron con los requisitos de poseer una carga factorial de .400 o superior. Se muestra la distribución de los reactivos en cada uno de los seis factores del instrumento que cumplieron con el requisito de poseer un valor Eigen de 1 o superior. Un total de 35 reactivos cumplieron con los requisitos mencionados ( $\mathrm{y}$ con no poseer correlaciones negativas ítem-instrumento).

Tabla 1. Valores Eigen y porcentaje de varianza del SEEU-R

\begin{tabular}{c|c|c|c|c} 
Factor & Reactivos & Valor Eigen & \% de varianza & $\begin{array}{c}\text { \% de varianza } \\
\text { acumulada }\end{array}$ \\
\hline 1 & 8 & 4.49 & 12.83 & 12.83 \\
\hline 2 & 10 & 3.95 & 11.29 & 24.19 \\
\hline 3 & 7 & 3.82 & 10.91 & 35.04 \\
\hline 4 & 4 & 3.03 & 8.66 & 43.71 \\
\hline 5 & 3 & 1.92 & 5.47 & 49.19 \\
\hline 6 & 3 & 1.70 & 4.87 & 54.06 \\
\hline
\end{tabular}

Alfa $=.931$ 
Tabla 2. Solución factorial rotada del SEEU-R

\begin{tabular}{|c|c|c|c|c|c|}
\hline Factor 1 & Factor 2 & Factor 3 & Factor 4 & Factor 5 & Factor 6 \\
\hline Ítem/ Peso & Ítem/ Peso & Ítem/ Peso & Ítem/ Peso & Ítem/ Peso & Ítem/ Peso \\
\hline $34 / .675$ & $12 / .708$ & $14 / .689$ & $27 / .703$ & $3 / .644$ & $10 / .675$ \\
\hline $21 / .663$ & $18 / .645$ & $16 / .663$ & $28 / .683$ & $5 / .552$ & $22 / .613$ \\
\hline $20 / .635$ & $13 / .603$ & $7 / .646$ & $26 / .622$ & $4 / .520$ & $35 / .518$ \\
\hline $19 / .588$ & $9 / .575$ & $2 / .556$ & $17 / .573$ & & \\
\hline $31 / .581$ & $32 / .531$ & $25 / .556$ & & & \\
\hline $29 / .571$ & $24 / .531$ & $11 / .540$ & & & \\
\hline $1 / .550$ & $33 / .528$ & $8 / .534$ & & & \\
\hline $23 / .478$ & $\begin{array}{c}6 / .504 \\
30 / .502 \\
15 / .458\end{array}$ & & & & \\
\hline
\end{tabular}

Tabla 3. Reactivos por factor

\begin{tabular}{l|l} 
Factor & Reactivos que constituyen al factor \\
\hline 1 & $\begin{array}{l}\text { R. 34. ¿En periodos de mucho estrés ha sufrido problemas relacionados con su pensamiento } \\
\text { (falta de atención, memoria, concentración)? } \\
\text { R. 21. ¿Ha sufrido hábitos relacionados al estrés (morderse las uñas, rechinar los dientes, } \\
\text { tronarse los dedos, etc.)? } \\
\text { R. 20. ¿Ha sufrido enfermedades directamente vinculadas al estrés (gastritis, migraña, úlceras, } \\
\text { dermatitis, etc.)? }\end{array}$ \\
$\begin{array}{l}\text { R.19. ¿Ha notado que su estado de ánimo cambia (irritabilidad, tristeza, agresividad) por la } \\
\text { exigencia académica y de trabajo de la universidad? }\end{array}$ \\
$\begin{array}{l}\text { R. } 31 . \text { ¿El pensar en sus calificaciones/tareas/exámenes, ¿le cambia el ánimo (mal humor, } \\
\text { depresión, ansiedad, preocupación, volubilidad, etc.)? }\end{array}$ \\
$\begin{array}{l}\text { R. 29. ¿Ha sufrido crisis nerviosas (ataques de pánico, taquicardia, hiperventilación, sudoración } \\
\text { de manos, etc.) atribuidas al exceso de trabajo, durante el periodo escolar? }\end{array}$ \\
R.1. ¿Ha tenido ansiedad intensa? \\
R. 23. ¿Ha sentido que sus problemas no tienen una solución?
\end{tabular}




\begin{tabular}{|c|c|}
\hline Factor & Reactivos que constituyen al factor \\
\hline 2 & $\begin{array}{l}\text { R.12. ¿Sus compañeros se han burlado de usted por sus calificaciones? } \\
\text { R.18. ¿Siente que su nivel académico está por debajo del de sus compañeros? } \\
\text { R.13. ¿Los profesores le han hecho comentarios negativos por su desempeño académico o } \\
\text { calificaciones? } \\
\text { R. 9. ¿Otras personas lo presionan a mejorar su promedio o desempeño escolar? } \\
\text { R. 32. ¿Ha pensado cambiar de carrera por el exceso de trabajo? } \\
\text { R. 24. ¿En trabajos en grupo frecuentemente se preocupa de no poder mantener el ritmo de } \\
\text { sus compañeros? } \\
\text { R. 33. ¿Ha tenido problemas familiares por su rendimiento académico (discusiones, castigos, } \\
\text { pleitos, etc.)? } \\
\text { R. 6. ¿Ha pensado en darse de baja por el exceso de trabajo escolar? } \\
\text { R. 30. ¿Ha decidido dejar una materia por el exceso de trabajo (reprobar a propósito, dar de } \\
\text { baja, etc.)? } \\
\text { R. 15. ¿Toma antidepresivos y/o ansiolíticos? }\end{array}$ \\
\hline 3 & $\begin{array}{l}\text { R. 14. ¿Se preocupa por alcanzar cierto nivel académico? } \\
\text { R. 16. ¿La exigencia de sus docentes es elevada? } \\
\text { R. 7. ¿Ha tenido una carga de trabajo excesiva? } \\
\text { R. 2. ¿Se ha desvelado por exámenes o trabajos? } \\
\text { R. 25. ¿Durante el semestre ha sentido que necesita unas vacaciones? } \\
\text { R. 11. ¿Tiene tanto trabajo que no sabe ni por dónde empezar? } \\
\text { R. 8. ¿Ha sentido ansiedad debido a las calificaciones que obtiene? }\end{array}$ \\
\hline 4 & $\begin{array}{l}\text { R. 27. ¿Ha sentido que la universidad exige un nivel académico excesivamente alto? } \\
\text { R. 28. ¿Falta a clases porque se siente muy presionado por la universidad? } \\
\text { R. 26. ¿Siente que ir a clase es una obligación pesada y abrumadora? } \\
\text { R. 17. ¿Siente que el número de materias por semestre es excesivo? }\end{array}$ \\
\hline 5 & $\begin{array}{l}\text { R. 3. ¿Consume más cigarros, alcohol y/o drogas de lo habitual en periodos de exámenes? } \\
\text { R. 5. ¿Se enferma en periodos de exámenes? } \\
\text { R. 4. ¿Ha sufrido de trastornos del sueño (insomnio, dormir demasiado, etc.) en periodos de } \\
\text { exámenes? }\end{array}$ \\
\hline 6 & $\begin{array}{l}\text { R.10. ¿Además de sus responsabilidades académicas ha tenido otras? (trabajo, cuidar algún } \\
\text { familiar, deporte de alto rendimiento, etc.) } \\
\text { R. 22. ¿Ha sufrido alguna pérdida significativa ya sea familiar, emocional y/o material en el } \\
\text { último año? } \\
\text { R. } 35 \text {. ¿Está atravesando por una situación difícil (enfermedad, crisis económica, separación, } \\
\text { mudanza, etc.)? }\end{array}$ \\
\hline
\end{tabular}


La tabla 3 muestra los reactivos que constituyen cada factor, se presentan ordenados con base al valor numérico de su carga factorial, presentada en la tabla 2 .

Una vez determinados los factores que constituyen al SEEU, se realizó un análisis de los diferentes aspectos que evalúan los reactivos constituyentes de cada factor. Para este proceso se imprimieron los reactivos y fueron analizados por tres jueces expertos en elaboración de instrumentos. Los jueces analizaron la agrupación de reactivos y buscaron los aspectos comunes, así como una etiqueta definitoria. Los resultados se presentan en la tabla 4.

Tabla 4. Dimensiones que evalúa cada factor

\begin{tabular}{c|l} 
Factor & Dimensiones que evalúa el factor \\
\hline 1 & $\begin{array}{l}\text { Somatización y ansiedad: Mide cambios en la salud inducidos por el estrés; también refiere a ansiedad } \\
\text { extrema inducida por estrés. }\end{array}$ \\
\hline 2 & $\begin{array}{l}\text { Estrés inducido por terceros: Mide el malestar que provocan profesores, compañeros y familiares sobre } \\
\text { el estudiante, en su empeño porque éste alcance un cierto rendimiento académico. }\end{array}$ \\
\hline 3 & $\begin{array}{l}\text { Estrés inducido por carga de trabajo: Mide el malestar que provocan en el estudiante los requisitos de } \\
\text { acreditación institucionales (exámenes, trabajos, tareas, etc.). }\end{array}$ \\
\hline 4 & $\begin{array}{l}\text { Desmotivación: Mide el grado en que el estrés académico excesivo ha llevado al estudiante a perder el } \\
\text { interés por sus estudios universitarios. }\end{array}$ \\
\hline 5 & Efectos colaterales: Mide daños en la salud del estudiante ocasionados por el estrés. \\
\hline 6 & Eventos de vida: Mide la presencia de eventos estresantes ajenos a la universidad. \\
\hline
\end{tabular}

Se calcularon correlaciones de Pearson entre los puntajes obtenidos en el SEEU, los puntajes obtenidos en el BDI y el BAI. Los resultados se muestran en la tabla 5.

Los análisis de inferencia estadística mostraron que las mujeres obtienen puntajes significativamente más altos que los hombres en el SEEU ( $\mathrm{t}(963)=3.033$, $\mathrm{p}<.001)$.

Con la finalidad de elaborar la versión de 18 reactivos del SEEU (SEEU-RR) se tomaron 18 reactivos de los seis factores identificados en el análisis factorial exploratorio. De cada área se tomaron los reactivos que alcanzaron las cargas factoriales más altas. La tabla 6 muestra los reactivos que se tomaron de cada factor.
Tabla 5. Coeficientes de correlación de Pearson

\begin{tabular}{c|c|c} 
Variable & BDI & BAI \\
\hline SEEU-R & $(r(970)=.431, p<.001)$ & $(r(970)=.347, p<.001)$ \\
\hline
\end{tabular}

Tabla 6. Reactivos del SEEU-RR

\begin{tabular}{c|c} 
Factor & Reactivos \\
\hline 1 & $34,21,20,19,12$ \\
\hline 2 & $18,13,9$ \\
\hline 3 & $14,16,7,2$ \\
\hline 4 & 27,28 \\
\hline 5 & 3,5 \\
\hline 6 & 10,22 \\
\hline
\end{tabular}


Tabla 7. Coeficientes de correlación de Pearson

\begin{tabular}{c|c|c|c} 
Variable & BDI & BAI & SEEU-R \\
\hline SEEU-RR & $(r(970)=.387, p<.001)$ & $(r(970)=.311, p<.001)$ & $(r(970)=.974, p<.001)$
\end{tabular}

Tabla 8. Estadísticos descriptivos

\begin{tabular}{c|c} 
SEEU-R & SEEU-RR \\
\hline $25 \%=25$ & $25 \%=15$ \\
\hline $50 \%=37$ & $50 \%=22$ \\
\hline $75 \%=54$ & $75 \%=31$ \\
\hline Media $=39.96, \mathrm{DE}=19.83$ & Media $=23.34, \mathrm{DE}=11.04$ \\
\hline
\end{tabular}

Tabla 9. Reactivos con frecuencias más altas para los últimos 30 días

\begin{tabular}{l|c} 
Reactivo & Frecuencia / $\%$ \\
\hline R. 2. ¿Se ha desvelado por exámenes o trabajos? & $377 / 37.8 \%$ \\
\hline R. 4. ¿Ha sufrido trastornos de sueño en periodos de exámenes? & $278 / 27.9 \%$ \\
\hline R. 7. ¿Ha tenido una carga de trabajo excesiva? & $277 / 27.8 \%$ \\
\hline R. 14. ¿Se preocupa por alcanzar un cierto nivel académico? & $324 / 32.5 \%$ \\
\hline R. 21. ¿Ha presentado conductas relacionadas con el estrés? & $285 / 28.6 \%$ \\
\hline R. 25. ¿Durante el semestre ha sentido que necesita vacaciones? & $391 / 39.2 \%$ \\
\hline
\end{tabular}

La tabla 7 muestra las correlaciones entre el SEEURR, el BDI, el BAI y las correspondientes al SEEU-R.

El SEEU-RR mostró una consistencia interna aceptable (.880).

Con la finalidad de iniciar el desarrollo de normas de calificación para el SEEU-R y el SEEU-RR, se calcu- laron los cuartiles para cada instrumento; además se calculó la media aritmética y desviación estándar de cada instrumento. La tabla 8 muestra los resultados.

La tabla 9 muestra los reactivos que tuvieron una frecuencia más alta de respuestas positivas en los últimos 30 días. 


\section{DISCUSIÓN}

Los datos obtenidos en la investigación coinciden con los producidos por Pulido et. al. (2011), en el sentido de que el instrumento se subdivide en seis factores independientes. Algunos de los factores identificados coinciden con las categorías planteadas por Pulido y colaboradores (op. cit.). Por ejemplo, el factor de "somatización y ansiedad" es muy similar al de "presencia de síntomas" identificado por Pulido y colegas. Complementariamente, tanto en el presente estudio como en el de Pulido et al., existe un factor que mide "carga académica" y otro que mide "desmotivación”. Además, en ambos estudios aparece un factor que considera el efecto que tienen terceros sobre el estrés académico del estudiante. A diferencia del estudio de Pulido y colaboradores, en la presente investigación los "eventos de vida" aparecieron como un factor independiente. Los reactivos relacionados con "estrategias para disminuir los efectos del estrés académico" no se agruparon en un factor independiente.

En lo concerniente a la validez empírica del estudio, los resultados replican hallazgos bien conocidos en el área. El SEEU-R y el SEEU-RR mantienen correlaciones directas y estadísticamente significativas con ansiedad; el hallazgo coincide con los estudios de Eisenberg et al. (2007); Leung, Yeung y Wong (2010); Misra y McKean (2000) y Rawson, Bloomer y Kendall (1994). Además, el SEEU-R y el SEEU-RR mantienen correlaciones directas y estadísticamente significativas con depresión; este resultado coincide con el reportado por Ang y Huan (2006); Bayram y Bilgel (2008); Mahmoud et al., (2012), entre otros. La investigación sobre estrés académico también ha reportado, de manera consistente, que las mujeres son más susceptibles que los hombres a este padecimiento (Brougham, Zail, Mendoza \& Miller, 2009; Huan, Yeo, Ang \& Chong, 2006; Ptacek, Smith \& Dodge, 1994); en la presente investigación también ocurrió así.

En lo concerniente a la confiabilidad de los instrumentos, tanto el SEEU-R como el SEEU-RR mostraron valores alfa de Cronbach aceptables (Nunnally, 1987). Los valores obtenidos son, además, muy similares a los reportados por Pulido y colaboradores en su trabajo seminal.

En síntesis, las dos versiones cortas del SEEU evaluadas en este estudio parecen tener propiedades psi- cométricas aceptables. Así, pues, se cuenta con instrumentos válidos y confiables desarrollados en muestras de estudiantes mexicanos. Dados los efectos del estrés académico sobre la salud y rendimiento de los estudiantes, tal parece que emplear estas escalas (u otras) en instituciones educativas, de manera habitual parece recomendable (Hystad et al., 2009; Watson, 2012). Hacerlo podría tener dos efectos educativos deseables: por un lado permitiría identificar estudiantes que requieren atención psicopedagógica de manera inmediata. Por otro, permitiría a la institución hacer ajustes en sus programas, para que éstos regulen su demanda académica de una forma "amigable" con la salud de sus estudiantes. Evaluar estrés académico ( $\mathrm{y}$ otros indicadores de salud universitaria) de manera continua y periódica, podría ser un hábito recomendable para una institución educativa; es decir, los datos empíricos cuantitativos cobran mayor valor para la toma de decisiones estratégicas cuando se observan a lo largo del tiempo, cuando es posible identificar tendencias y patrones (Pulido, 2010). Los datos cobran más utilidad cuando grupos de instituciones educativas emplean los mismos instrumentos de manera sincronizada. $\mathrm{Al}$ comparar entre instituciones es posible aproximarse a parámetros de "normalidad/anormalidad". Además, es posible valorar los efectos que las variables ecológicas, políticas o educativas tienen sobre la salud de las comunidades estudiantiles (Pulido et al., 2014; Schonlau et al., 2008). Hasta donde los autores saben, en México no existen instituciones que evalúen de manera sistemática y periódica el estrés académico de los estudiantes. Si esto se debe a la ausencia de instrumentos con propiedades psicométricas conocidas, el presente estudio procuró remediar dicha carencia.

En cuanto al significado teórico de los resultados, el carácter correlacional del diseño impide determinar relaciones de causa-efecto. La interpretación tradicional del hallazgo concluye que los eventos de vida estresantes deterioran la salud física y mental de los individuos (Holmes y Rahe,1967). Desde los modelos cognitivos, los eventos de vida estresantes son mediados por la interpretación que hace el sujeto de los mismos eventos; sin embargo, también son conceptualizados como agentes casuales indirectos de la ansiedad y de la depresión (Beck, 1976). La idea de que 
el estrés académico produce ansiedad y depresión ha sido apoyada por revisiones del área (Putwain, 2007) y por análisis de regresión (Ang \& Huan, 2006).

En lo concerniente a las diferencias por género encontradas en este estudio ( $\mathrm{y}$ en otros), la interpretación más habitual ha sido la de que hombres y mujeres emplean diferentes estrategias para enfrentar el estrés académico. Específicamente, parece que las mujeres emplean estrategias "emocionales" y los hombres estrategias de "solución de problemas" (Brougham et al., 2009).

\section{REFERENCIAS}

Ang, R. P. \& Huan, V. S. (2006). Academic expectations stress inventory (AESI): Development, factor analysis, reliability and validity. Educational and Psychological Measurement, 66, 522-539. Recuperado de http://dx.doi.org/10.1177/0013164405282461

Bayram, N. \& Bilgel, N. (2008). The prevalence and sociodemographic correlations of depression, anxiety and stress among a group of university students. Social Psychiatry and Psychiatric Epidemiology, 43, 672-677. Recuperado de http://dx.doi.org/10.1007/s00127008-0345-x

Beck, A. T. (1976). Cognitive therapy and the emotional disorders. New York, Meridian.

Brougham, R. R., Zail, C. M., Mendoza C. M. \& Miller J, R. (2009). Stress, sex differences, and coping strategies among college students. Current Psychology, 28, 85-97. Recuperado de http://link.springer.com/ article/10.1007\%2Fs12144-009-9047-0

Chang, E. C. (2001). Life stress and depressed mood among adolescents: examining a cognitive-affective mediation model. Journal of Social and Clinical Psychology, 20, 416-429. Recuperado de http://dx. doi.org/10.1521/jscp.20.3.416.22301

Connor, M. J. (2001). Pupil stress and standard assessment tests (SATS). Emotional and Behavioural Difficulties, 6, 103-111. Recuperado de http://dx.doi. org/10.1080/13632750100507660

Connor, M. J. (2003) Pupil stress and standard assessment tests (SATS): an update, Emotional and Behavioural Difficulties, 8, 101-107. Recuperado de http://dx.doi. org/10.1177/1363275203008002002

Dyson, R. \& Renk, K. (2006). Freshmen adaption to university life: Depressive symptoms, stress and
Es decir, las primeras se centran en expresar su malestar, los segundos diseñan planes y estrategias de afrontamiento (Eaton \& Bradley, 2008). El afrontamiento centrado en la solución de problemas se encuentra asociado con menores niveles de estrés académico (Stanton, Kirk, Cameron y Danoff-Burg 2000). Estudios futuros permitirán evaluar esta hipótesis en estudiantes universitarios mexicanos y además podrán establecer relaciones causales entre las variables exploradas en esta investigación. •

coping. Journal of Clinical Psychology, 62, 1231-1244. Recuperado de http://dx.doi.org/10.1002/jclp.20295

Eaton, R. J. \& Bradley, G. (2008). The role of gender and negative affectivity in stressor appraisal and coping selection. International Journal of Stress Management, 15, 94-115. Recuperado de http:// dx.doi.org/10.1037/1072-5245.15.1.94

Edwards, K. J., Hershberger, P. J., Russell, R. K. \& Markert, R. J. (2001). Stress, negative social, exchange, and health symptoms in university students. Journal of American College Health, 50, 75-79. Recuperado de http://dx.doi.org/10.1080/07448480109596010

Eisenberg, D., Gollust, S. E., Golberstein, E., \& Hefner, J. L. (2007). Prevalence and correlates of depression, anxiety, and suicidality among university students. American Journal of Orthopsychiatry, 77, 534-542. Recuperado de http://dx.doi.org/10.1037/0002-9432. 77.4.534

Eremsoy, C. E., Çelimli, S \& Gençöz, T. (2005). Students under academic stress in a Turkish University: variables associated with symptoms of depression and anxiety. Current Psychology, 24, 123-133. Recuperado de http://dx.doi.org/10.1007/s12144-005-1011-z

Field, A. (2005). Discovering statistics using SPSS. Londres, Inglaterra: Sage.

García-Ros, R., Pérez-González, F., Pérez-Blasco, J., \& Natividad, L.A. (2012). Evaluación del estrés académico en estudiantes de nueva incorporación a la universidad. Revista Latinoamericana de Psicología, 44, 143-154.

Gloria, A. M. \& Ho, T. A. (2003). Environmental, social, and psychological experiences of Asian-American under-graduates: examining issues of academic per- 
sistence. Journal of Counseling and Development, 81, 93-106. Recuperado de http://dx.doi.org/10.1002/j. 1556-6678.2003.tb00230.x

Holmes, T. H. y Rahe, R. H. (1967). The social readjustment scale. Journal of Psychosomatic Research, 11, 213-221.

Hudd, S., Dumlao, J., Erdmann-Sager, D., Murray, D., Phan, E., Soukas, N. \& Yokosuka, N. (2000). Stress at college: effects on health habits, health status and self-esteem. College Student Journal, 34, 217-227.

Hystad, S. W., Eid, J., Laberg, J. C. y Johonsen, B. H. (2009). Academic stress and health: Exploring the moderating role of personality hardiness. Scandinavian Journal of Educational Research, 53, 421-429. Recuperado de http://dx.doi.org/10.1080/003138309 03180349

Ho B. K. W., Hong, C. \& Kua, E. H. (1999). Suicidal behavior among young people in Singapore. General Hospital Psychiatry, 21, 128-133. Recuperado de http://dx.doi.org/10.1016/s0163-8343(98)00065-6

Huan, V. S., Yeo S, L., Ang, R. P. \& Chong, H. W. (2006). The influence of dispositional optimism and gender on adolescent's perception of academic stress. Adolescence, 41, 533-546. Recuperado de http://dx. doi.org/10.1007/s12144-006-1004-6

Jurado, S., Villegas, M. E., Méndez, L., Rodríguez, F., Loperena, V. y Varela, R. (1998). La estandarización del Inventario de Depresión de Beck para los residentes de la ciudad de México. Salud Mental, 21, 26-31.

Leung, G. S. M., Yeung, K. C. \& Wong, D. F. K. (2010). Academic stressors and anxiety in children: the role of paternal support. Journal of Child and Family Studies, 19, 90-100. Recuperado de http://dx.doi. org/10.1007/s10826-009-9288-4

Lumley, M. A. \& Provenzano, K. M. (2003). Stress management through written emotional disclosure improves academic performance among college students with physical symptoms. Journal of Educational Psychology, 95, 641-649. Recuperado de http://dx.doi. org/10.1037/0022-0663.95.3.641

Mahmoud, J. S. R., Staten, R., Hall, L. A. \& Lennie, T.A. (2012). The relationship among young adult college students' depression, anxiety, stress, demographics, life satisfaction, and coping styles. Issues in Mental Health Nursing, 33, 149-156. Recuperado de http:// dx.doi.org/10.3109/01612840.2011.632708
Mc Donald, A. S. (2001). The prevalence and affects of test anxiety in school children. Educational Psychology, 21, 89-101. Recuperado de http://dx.doi.org/ $10.1080 / 01443410020019867$

Misra, R. \& McKean, M. (2000). College student's academic stress and its relation to their anxiety, time management, and leisure satisfaction. American Journal of Health Studies, 16, 41-51.

Misra, R., Mckean, M., West, S. \& Russo, T. (2000). Academic stress of college students: comparison of student and faculty perceptions. College Student Journal, 34, 236-245.

Nunnally, J. (1978). Psychometric theory. New York: McGraw-Hill.

Polo, A., Hernández, J. M. y Poza, C. (1996). Evaluación del estrés académico en estudiantes universitarios. Ansiedad y Estrés, 2, 159-172.

Ptacek, J. T., Smith, R. E. \& Dodge, K.L. (1994). Gender differences in coping with stress: when stressor and appraisals do not differ. Personality and Social Psychology Bulletin, 20, 421-430. Recuperado de http://dx.doi.org/10.1177/0146167294204009

Pulido, M. (2010). Observatorio de salud universitaria de la UIC. Revista Foro UIC, 17, 61-64.

Pulido, M., Escoto, R. \& Gutiérrez, D. M. (2011). Validez y confiabilidad del cuestionario de uso problemático de internet. Journal of Behavior, Health and Social Issues, 3, 25-34.

Pulido, M., Vázquez, M. A., García, M., Guillot, C., Morales, J. A., Moreno, P., Moranchel, J. A., Portilla, D., Rodríguez, P., Rodríguez, G. y Sosa, J. (2014). Variables ecológicas y consumo de sustancias adictivas en universitarios de la Ciudad de México: historia de dos universidades. Revista Intercontinental de Psicología y Educación. En prensa.

Putwain, D. (2007). Researching academic stress and anxiety in students: some methodological considerations. British Educational Research Journal, 33, 207-219. Recuperado de http://dx.doi.org/10.1080/ 01411920701208258

Rawson, H. E., Bloomer, K., \& Kendall, A. (1994). Stress, anxiety, depression, and physical illness in college students. The Journal of GeneticPsychology, 155, 321330. Recuperado de http://dx.doi.org/10.1080/00221 325.1994 .9914782 
Schonlau, M., Scribner, R., Farley, T., Theall, K.P., Bluthenthal, R. N., Scott, M. \& Cohen, D. A. (2008). Alcohol outlet density and alcohol consumption in Los Angeles County and Southern Louisiana. Geospatial Health, 3, 91-101.

Stanton, A. L., Kirk, S. B., Cameron, C., \& Danoff-Burg, S. (2000). Coping though emotional approach: scale construction and validation. Journal of Personality and Social Psychology, 78, 1150-1169. Recuperado de http://dx.doi.org/10.1037//0022-3514.78.6.1150

Sue, S. \& Okazaki, S. (1990). Asian-American educational achievements: a phenomenon in search of an explanation. American Psychologist, 45, 913-920. Recuperado de: http://dx.doi.org/10.1037//0003-066x. 45.8.913
Tafoya, R., Gómez, P., Ortega, S. y Ortiz, L. S. (2006). Inventario de ansiedad de Beck: validez y confiabilidad en estudiantes que solicitan atención psiquiátrica en la UNAM. Psiquis, 15, 82-87.

Toero, K., Nagy, A., Sawaguchi, A. y Sotonnyi, P. (2001) Characteristics of suicide among children and adolescents in Budapest. Pediatrics International, 43, 368-371.

Ung, E.K. (2003). Youth suicide and parasuicide in Singapore. Annals of the Academy of Medicine of Singapore 32, 1-7.

Watson, J. C. (2012). Managing college stress: the role of college counselors. Journal of College Counseling, 15, 3-4. Recuperado de http://dx.doi.org/10.1002/ j.2161-1882.2012.00001. 